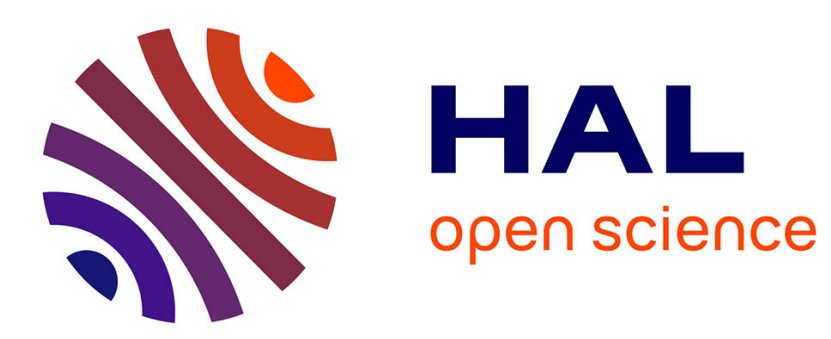

\title{
Retrodirective metasurfaces from non-reciprocal to reciprocal using impedance modulation for high-super-cell-periodicity designs
}

Mohammed Kalaagi, Divitha Seetharamdoo

\section{- To cite this version:}

Mohammed Kalaagi, Divitha Seetharamdoo. Retrodirective metasurfaces from non-reciprocal to reciprocal using impedance modulation for high-super-cell-periodicity designs. Applied physics. A, Materials science \& processing, 2020, 126 (4), pp.300. 10.1007/s00339-020-3455-2 . hal-02925049

\author{
HAL Id: hal-02925049 \\ https://hal.science/hal-02925049
}

Submitted on 28 Aug 2020

HAL is a multi-disciplinary open access archive for the deposit and dissemination of scientific research documents, whether they are published or not. The documents may come from teaching and research institutions in France or abroad, or from public or private research centers.
L'archive ouverte pluridisciplinaire HAL, est destinée au dépôt et à la diffusion de documents scientifiques de niveau recherche, publiés ou non, émanant des établissements d'enseignement et de recherche français ou étrangers, des laboratoires publics ou privés. 


\title{
Retrodirective Metasurfaces from Non Reciprocal to Reciprocal Using Impedance Modulation for High Super-cell Periodicity Designs
}

\author{
Mohammed Kalaagi • Divitha Seetharamdoo
}

Received: date / Accepted: date

\begin{abstract}
The growing popularity of metasurfaces and their exceptional physical phenomenons, have gained remarkable interest in terms of propagation and electromagnetic wave control. Particularly, for the case of retro-reflection, retrodirective gradient metasurfaces are well known for their reciprocal responses in terms of retro-reflection specially for subwavelength super-cell periodicity designs. Recently, we have observed that for metasurfaces with high super-cell periodicities greater than $2 \lambda$, the retrodirective metasurface loses its reciprocal response in terms of retrodirectivity. The main challenge in this case, is to retain the reciprocal response, as well as achieving efficient responses for retroreflection at the higher orders of diffraction that exist at these periodicities. Thus, in this paper we demonstrate the design of a retrodirective metasurface from non reciprocal to reciprocal following the generalized phase law of reflection and introducing the impedance modulation technique, for a metasurface operating at 14.7 $\mathrm{GHz}$ with a super-cell periodicity of $2.88 \lambda$. On the other hand, with a well engineered surface impedance of a super-cell at these periodicities, efficient responses of retro-reflection can be achieved at the higher orders of diffraction, giving rise to a retrodirective metasurface design operating simultaneously for multiple incident angles. Retro-reflection has been achieved at eleven angles simultaneously and the reciprocal property was retained at the desired angle of incidence $10^{\circ}$.
\end{abstract}

M. Kalaagi

Tel.: +33641866027

E-mail: mohammad.kalaagi@railenium.eu · D. Seetharamdoo E-mail: divitha.seetharamdoo@ifsttar.fr

University of Lille 1 Nord de France, at IFSTTAR, COSYS, LEOST and the Institut de Recherche Technologique Railenium , F-59650 Villeneuve d'Ascq
Keywords Metasurfaces · Retro-reflection · Multiple angles · Surface Impedance Modulation · High Supercell periodicity designs

\section{Introduction}

Metasurfaces [1,2] have gained significant interest for their potential to manipulate the propagation of an electromagnetic wave in unconventional ways $[3,4]$. Many functional designs have been developed based on metasurfaces for different applications in terms of electromagnetic wave control, which include beam steering $[5-7]$ as well as the transformation of propagating waves into surface waves [8], multi-functional metasurfaces [9], polarization control [10], hologram [11], at microwave and optical frequencies. Different metasurface designs have also been introduced for dual polarization applications [12-14], as well as for broad band frequency designs $[15-17]$. In the context of wave manipulation and control based on metasurface designs, retrodirective metasurfaces [12] have been shown to be an interesting feature for different applications such as radar cross section (RCS) enhancement [18, 19], specially for radar targets where backscattered power reflection level is poor. It is well known that this phenomenon can be determined at normal incidence for a conventional metallic flat plate or mirror based on Snell's law.

In previous works, N. Yu et al. have proved that following the generalized phase law of reflection [20], i.e. by introducing a phase gradient between scatterers, the control of an EM wave in a desired direction can be achieved [3]. Doumanis et. al [21] have designed retrodirective reflectors following the reflect array approach. In their case, retrodirectivity was obtained for 
a single angle of incidence at subwavelength periodicity for the designed super-cell, as well as the reciprocal property of the design was present, thus validating the theory of the generalized phase law.

Indeed, retro-reflection can be achieved at a given incident angle, it is well known that it can be directly achieved in the opposite or reciprocal direction [3], [21], $[22]$. We recall in previous work the design of retrodirective metasurfaces for various angles of incidence at different super-cell periodicity designs [23]. It has been shown that, for retrodirective metasurface designs with subwavelength super-cell periodicities, the metasurface can perform retro-reflection for both the desired direction and the opposite reciprocal direction as well as at normal incidence [3,21-23]. However, according to Floquet-Bloch harmonic analysis [24], [25], with an increase of periodicity higher than the wavelength $\lambda$, higher orders of diffraction can exist for the super-cell design, which creates more apertures for wave propagation at different angles of incidence. For a supercell periodicity between $\lambda$ and $2 \lambda$, retro-reflection for the metasurface at normal incidence is shown to be lost [23]. On the other hand, for periodicities greater than $2 \lambda$, the metasurface loses its conventional reciprocal property for retro-reflection [23], which can be due to the existance of higher orders of diffraction and apertures where the wave is propagating. Recently, V.S. Asadchy et al. have introduced the concept of the surface impedance modulation [26], to design multi-channel reflectors [25], under the assumption of lossless and reciprocal reflectors at a periodicity of $1.064 \lambda$. Although this concept has been demonstrated for an incident angle of $28^{\circ}$, it can be interesting to study the design of retrodirective metasurfaces addressing lower incident angles such as $10^{\circ}$ where the periodicity can reach a level up to $2.88 \lambda$.

The main challenge is to retain the conventional reciprocal property of a retrodirective metasurface at high super-cell periodicity designs and achieve efficient retroreflection at the higher orders of diffraction. In contrast to previous work, the concept of cascading has been introduced [23], in order to optimize the association for several retrodirective metasurfaces without the loss of their performance. However, the metasurface [23] is considered bulky and of large dimensions. In this paper, we demonstrate the possibility to design a retrodirective metasurface with a high super-cell periodicity up to $2.88 \lambda$ using the surface impedance modulation technique, this is, by retaining its conventional reciprocal property at $10^{\circ}$ and normal incidence with high efficiency using a single designed super-cell. Furthermore, taking advantage of the high number of diffraction orders at these periodicities and with a proper engineered surface impedance, one can extend the retrodirective metasurface to operate at multiple incident angles simultaneously.

The paper is organized as follows. Section 2 defines the concept and design procedure of the retrodirective metasurface using the impedance modulation technique, as well as the principle of operation for the design. In section 3 we introduce the metasurface design and results. In section 4 measurements have been carried out in an anechoic chamber for the metasurface design operating at $14.7 \mathrm{GHz}$, and it is shown that the measurements and simulation results are in very good agreement.

\section{Concept and Metasurface Design Procedure Using Surface Impedance Modulation}

\subsection{Principle of Operation}

First let us define the principle of operation for the design of a gradient metasurface. The starting point is by defining a configuration following the generalized phase law of reflection [20]. Particularly, for the case of retro-reflection, the incident angle $\theta_{i}=-\theta_{r}$ where $\theta_{r}$ is the angle of reflection. To ensure control of the full wavefront of the propagating wave, the integration (from 0 to $2 \pi$ ) of the generalized phase law of reflection is applied. In this case the periodicity of the super-cell defines the full control of the propagating wave in a desired direction. Thus, for the case of retrodirectivity, the periodicity $\mathrm{L}_{x}$ is defined as follows:

$$
\mathrm{L}_{x}=\frac{\lambda}{2 \sin \left|\theta_{i}\right|}
$$

The performance of retro-reflection for the metasurface design depends directly on the relation between the periodicity $\mathrm{L}_{x}$ and the wavelength $\lambda$, where $\theta_{i}$ varies according to the incident angle desired for retro-reflection. In this case, since $\mathrm{L}_{x}$ is the periodicity of the super-cell design, thus it can only be a positive value. Hence, in theory the metasurface should perform retro-reflection for the desired angle $\theta_{i}$ and reciprocal angle $-\theta_{i}$ simultaneously. This has been proved in previous work at subwavelength periodicity designs $[3,21,22,25]$. However, with an increase of periodicity beyond $\lambda$, the performance of the retrodirective metasurface differs and shows direct relation between the periodicity $\mathrm{L}_{x}$ and wavelength $\lambda$ as shown in Fig. 1 . For a periodicity $\mathrm{L}_{x}$ $\leq \lambda$, retroreflection is obtained at the desired angle, reciprocal direction and normal incidence as shown in Fig. 1a. However, for $\lambda<\mathrm{L}_{x}<2 \lambda$, the metasurface 


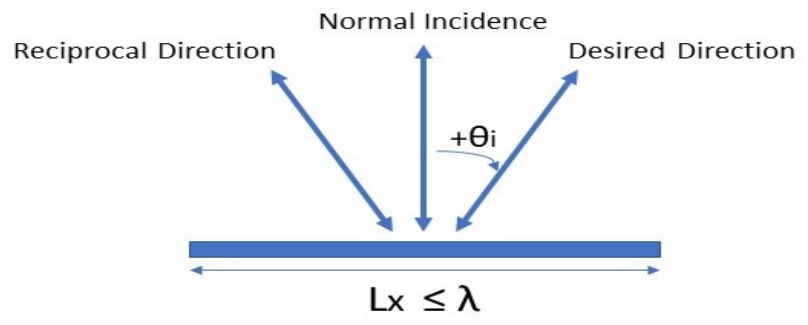

(a)

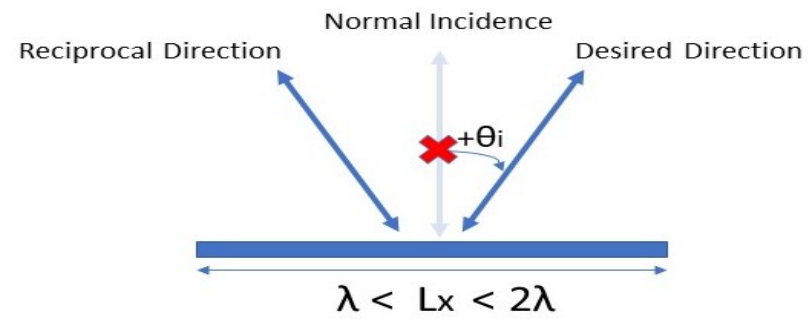

(b)

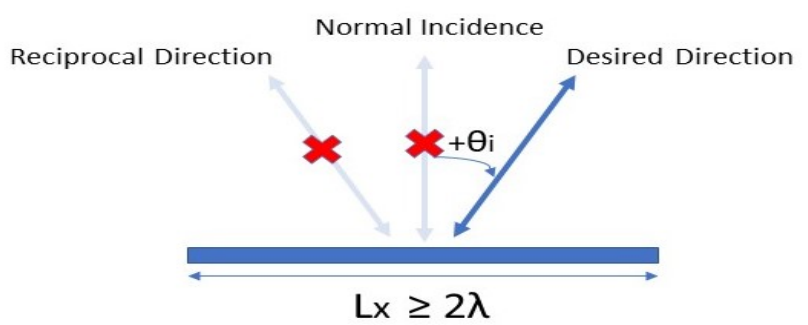

(c)

Fig. 1 a) Super-cell periodicity $\mathrm{L}_{x} \leq \lambda$, retro-reflection is obtained in the desired direction $\theta_{i}$, the reciprocal direction and at normal incidence. b) Super-cell periodicity $\lambda<\mathrm{L}_{x}<2 \lambda$, retro-reflection is obtained in the desired direction $\theta_{i}$ and the reciprocal direction, whereas for normal incidence is absent. c) Super-cell periodicity $\mathrm{L}_{x} \geq 2 \lambda$, retro-reflection is obtained only in the desired direction $\theta_{i}$, whereas the conventional properties of the metasurface are lost in the reciprocal and normal incidence directions.

loses its performance for retro-reflection at normal incidence [23] as shown in Fig. 1b. On the other hand, with an increase of periodicity beyond $2 \lambda\left(\mathrm{L}_{x} \geq 2 \lambda\right)$, the retrodirective metasurface loses its conventional reciprocal property and retroreflection is obtained only at the desired angle [23] as shown in Fig. 1c. According to Floquet-Bloch analysis [24], for a metasurface design of periodicity larger than $\lambda$, higher orders of diffraction and open apertures can exist. This can explain the loss of retro-reflection in the reciprocal direction and normal incidence, which is due to the the existence of open apertures which allow the propagation of the wave in other directions. However, the direction of the open apertures or higher orders of diffractions can be obtained as follows:

$\theta_{r n}=\arcsin \frac{n \lambda}{L_{x}}$

where $n$ defines the number of open apertures or diffraction orders and $\theta_{r n}$ the direction of the diffraction orders.

\subsection{High Periodicity Reciprocal Metasurface Design Procedure Using The Surface Impedance Modulation Technique}

The main challenge in this work is to retain the conventional reciprocal property for a retrodirective meta- surface as well as at normal incidence. However, following the surface impedance modulation technique, with a proper engineered surface impedance, for super-cell periodicities higher than $2 \lambda$, one can achieve efficient retro-reflection at the directions of the higher orders of diffraction. In this section, we explain briefly the design procedure of a reciprocal retrodirective metasurface for a periodicity design of $2.88 \lambda$ operating at $14.7 \mathrm{GHz}$. We define the nominal super-cell following the generalized phase law of reflection and the optimized super-cell design after introducing the surface impedance modulation technique.

The retrodirective metasurface consists of optimized super-cells which are designed in two steps. The starting point for the design of a gradient metasurface, is by defining a configuration based on the generalized phase law of reflection.The designed nominal super-cell in shown in Fig. 2.

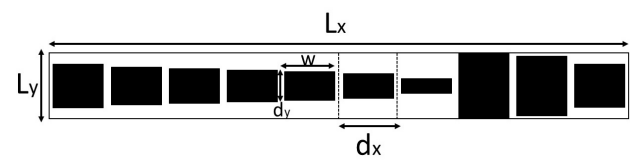

Fig. 2 Nominal super cell design discretized into 10 patch cells of dimensions $d_{y}$ (given in table 1 ) following the generalized phase law of reflection. 
The periodicity $\mathrm{L}_{x}$ and $\mathrm{L}_{y}$ of the nominal super-cell define the length and width of the super-cell respectively as shown in Fig. 2. $\mathrm{L}_{x}$ is determined according to Eq. (1). The patch dimensions $d_{y}$ are determined following the phase of the reflection coefficient which are tuned with respect to a phase gradient. In the second step the surface impedance optimization of the nominal super-cell design is implemented. It has been shown [26] that in order to ensure efficient retro-reflection of the designed metasurface, the surface impedance $Z_{s i}$ should be purely reactive at each patch element $i$ with respect to the given periodicity, where the incident electric field $\mathrm{E}_{i}$ is equal to that of the reflected electric field $\mathrm{E}_{r}$ [25], [26]. Assuming a given TM polarized wave $\mathrm{Z}_{T M}=\eta \cos \theta_{i}, Z_{s i}$ is given as:

$Z_{s i}=j \frac{Z_{T M}}{\tan \left(\frac{\phi_{i}}{2}\right)}$

Eq. (3) defines the surface impedance $Z_{\text {si }}$ needed for each patch element where $\phi_{i}$ defines the phase of the reflection coefficient. To achieve a purely reactive impedance of the super-cell design, post processing optimization using Floquet Periodic Boundary condtion of the nominal super-cell patch dimensions is followed. The process is done by tuning the nominal patch dimensions $d_{y}$ to obtain the best combination with a purely reactive surface impedance. Fig . 3 shows the super-cell design after numerical optimization of the patch cells to a proper reactive surface impedance.

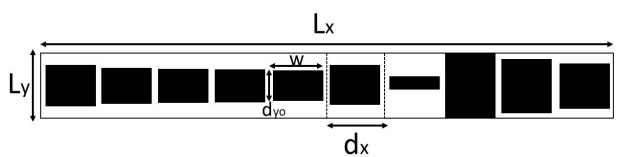

Fig. 3 Optimized Super-cell after tuning the patches to the proper reactive surface impedance.

The nominal and optimized super-cells are divided into $\mathrm{N}$ unit cells where $\mathrm{N}=10$ in this case, $d_{x}=$ $\mathrm{L}_{x} / N$, with the width of the patches being constant $w=d_{x}-0.5 \mathrm{~mm} . d_{y}$ defines the dimension of the nominal patches which are tuned with respect to a phase gradient. The dimensions of the patches are determined following the phase of the reflection coefficient $\phi_{i}$ shown in Fig .4.

For a given frequency of $14.7 \mathrm{GHz}$ and $\theta_{i}=10^{\circ}$, a progressive phase shift equal to $40.5^{\circ}$ is introduced between each patch element, to achieve the whole phase distribution given in Fig. 4 for the super-cell design. The periodicity $\mathrm{L}_{x}=2.88 \lambda$ and $\mathrm{L}_{y}=\lambda / 2$. Table 1 shows the nominal dimensions of the metallic patches with

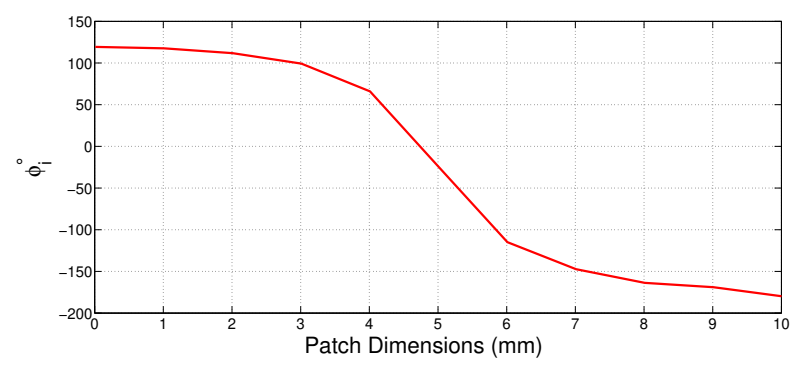

Fig. 4 Phase of the reflection coefficient with respect to the patch dimensions $d_{y}$ in mm for a given frequency at $14.7 \mathrm{GHz}$

respect to $\phi_{i}$, which are printed on a grounded substrate of permitivitty $\epsilon=2.2, \tan (\delta)=1 \times 10^{-3}$ and thickness $1.57 \mathrm{~mm}$ as shown in Fig.2.

For better understanding of the reciprocal property loss of the nominal supêr-cell design, the nominal surface impedance has been calculated with respect to the patch dimensions $d_{y}$ as shown in Fig .5. We can observe that the real part of the nominal surface impedance is a large positive value and indicates that the design is a lossy metasurface. We can also notice the imaginary part of $\mathrm{Z}_{s}$, which is not symmetric in this case for the negative and positive values at a reference value of zero. This also ensures that the reciprocal property of the metasurface design is absent for high periodicity supercell designs above $2 \lambda$. To overcome this problem and retain the conventional properties of the retrodirective metasurface the surface impedance is optimized to a purely reactive value as explained previously.

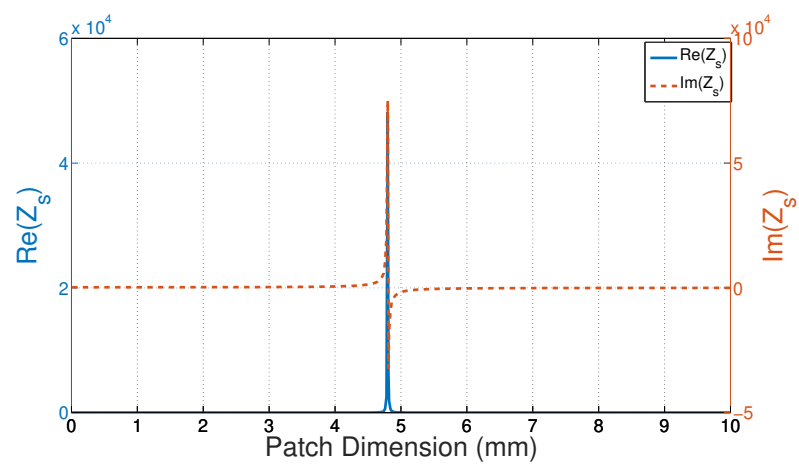

Fig. 5 The real and imaginary part of the surface impedance $\left(\operatorname{Re}\left(Z_{s}\right)\right.$ and $\left.\operatorname{Im}\left(Z_{s}\right)\right)$ with respect to the patch dimensions $d_{y}$ of the designed super-cell of periodicity $2.88 \lambda$.

Thus, the surface impedance of the nominal supercell shown in Fig.2 has been calculated at the given frequency $14.7 \mathrm{GHz}$ and incident angle $10^{\circ}$ for a $\mathrm{TM}$ polarized wave where $\mathrm{Z}_{s}=11.5-j 524.25$ using Floquet Periodic Boundary Condition. The patches are tuned to a level where the real part of $\mathrm{Z}_{s}$ is approximately equal to zero, this is determined by post processing numerical 
Table 1 The nominal dimensions $d_{y}(\mathrm{~mm})$ of the patch cells following the generalized phase law and the phase of the reflection coefficient $\phi_{i}{ }^{\circ}$ shown in Fig. 4 with a phase gradient equal to $40.5^{\circ}$, as well as the optimized dimensions $d_{y o}$ after implementation of the surface impedance modulation.

\begin{tabular}{lcr}
$\phi_{i}{ }^{\circ}$ & $\begin{array}{c}\text { Nominal } \\
\text { Dimensions } d_{y}(\mathrm{~mm})\end{array}$ & $\begin{array}{r}\text { Optimized } \\
\text { Dimensions } d_{\text {yo }}(\mathrm{mm})\end{array}$ \\
\hline-134.27 & 6.73 & 6.38 \\
-93.7 & 5.88 & 5.61 \\
-53.2 & 5.408 & 5.306 \\
-12.7 & 4.969 & 5.102 \\
27.8 & 4.489 & 4.898 \\
68.3 & 3.979 & 5.915 \\
108.8 & 2.306 & 2.04 \\
-210.7 & 10.2 & 10.2 \\
-170.2 & 9.28 & 8.673 \\
-129.7 & 6.53 & 6.73
\end{tabular}

optimization using Ansys HFSS and finding the best combination between the designed grounded patches for the super-cell design using Floquet boundary conditions. The optimized dimensions $d_{y o}$ have been given in Table 1. The surface impedance of the optimized super-cell design in Fig.3 has been optimized to a value of $\mathrm{Z}_{s}=0.9-j 267.3$ at $14.7 \mathrm{GHz}$, where the real part is approximately equal to zero thus eliminating the ohmic losses of the designed super-cell.

\section{Metasurface Design and Results}

Two metasurfaces have been designed with similar dimensions $5 \mathrm{~cm} \times 23 \mathrm{~cm}$ using Ansys HFSS; The first one of $5 \times 4$ nominal super-cells(Fig. 2) as shown in Fig. $6 \mathrm{a}$ and the second using $5 \times 4$ optimized super-cells(Fig. 3) given in Fig. 6b.

a)

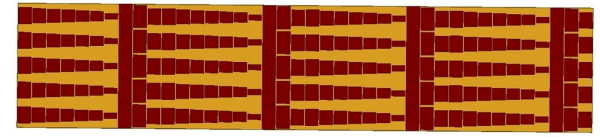

b)

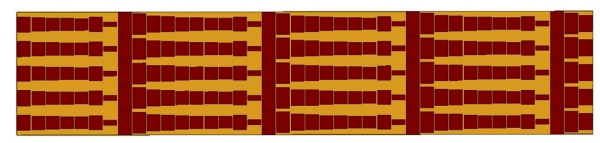

Fig. 6 a) Metasurface design using Ansys HFSS of $5 \times 4$ nominal super-cells b) Metasurface design of $5 \times 4$ optimized supercells .

To compare the performances of both metasurfaces, the normalized monostatic RCS is determined to calculate the energy which is redirected back to the direction of the transmitter as shown in Fig.7.

First for the case of the nominal or initial metasurface design in Fig. 6a, the results in Fig.7 show a high a level of retro-reflection for the nominal metasur-

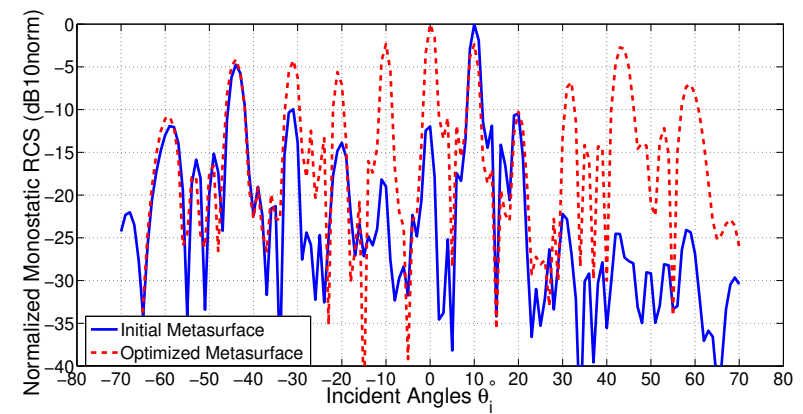

Fig. 7 Normalized Monostatic RCS of the optimized metasurface (Fig. 6b) after implementation of the impedance modulation compared to that of the nominal design (Fig. 6a) following the generalized phase law of reflection operating at 14.7 GHz and of similar dimensions $5.1 \mathrm{~cm} \times 23.5 \mathrm{~cm}$.

face at $10^{\circ}$ due to the proper periodicity and desired angle chosen. Other peaks in terms of retro-reflection are present but with low efficiency compared to the desired angle of incidence $\left(10^{\circ}\right)$ such as, $-20^{\circ},-44^{\circ},-32^{\circ}$ and $-60^{\circ}$. This is expected, since according to Floquet Bloch analysis in Eq. 2, higher diffraction orders can exist and the direction of the apertures can be calculated. Thus, for $\mathrm{L}_{x}=2.88 \lambda$, the range of values of $n$ are limited to $n=0, \pm 1, \pm 2$. Hence, five open apertures or higher orders of diffraction are expected at $\theta_{i}=0$, $\pm 20.3^{\circ}, \pm 44^{\circ}$. The presence of two other higher orders of diffraction at $\pm 32^{\circ}, \pm 60^{\circ}$ can be due to the level of periodicity which is approximately equal to $3 \lambda$. However, we can observe that, the reciprocal direction at $-10^{\circ}$ and normal incidence are absent and of very low efficiencies, which is due to the propagation of the wave in the direction of the higher diffraction orders which are said to be uncontrollable.

Second for the case of the optimized metasurface design in Fig. 6b, after the implementation of the surface impedance modulation technique, we can observe from Fig.7 that the retro-reflection in the reciprocal direction at $-10^{\circ}$ and normal incidence have been retained and achieved with high efficiency. This is due to the control of the high diffraction orders by optimizing the surface impedance, as well as, retro-reflection was also obtained with higher efficiency compared to that of the nominal case, in the direction of the diffraction orders at $\pm 20.3^{\circ}, \pm 32^{\circ}, \pm 44^{\circ}$ and $\pm 60^{\circ}$. To determine the efficiency of retro-reflection for the optimized metasurface at the eleven angles of incidence, the metasurface is compared to that of a metallic flat plate of similar dimensions $5.1 \mathrm{~cm} \times 23.5 \mathrm{~cm}$, which well known for its high efficiency in terms of retro-reflection at normal incidence. The simulated results comparing both performances are shown in Fig.8. 


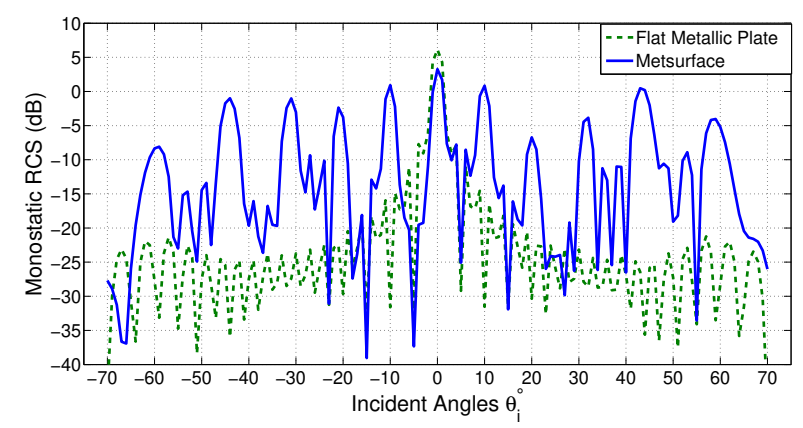

Fig. 8 Monostatic RCS (dB) of the optimized metasurface design(Fig. 6b) compared to that of flat metallic plate with respect to the given incident angles at $14.7 \mathrm{GHz}$ of similar dimensions $5.1 \mathrm{~cm} \times 23.5 \mathrm{~cm}$.

The results show a high level of monostatic RCS or retrodirectivity for the optimized metasurface design for the following incident angles at normal incidence and $\pm 10^{\circ}, \pm 20.3^{\circ}, \pm 32^{\circ}, \pm 44^{\circ}, \pm 60^{\circ}$ compared to that of the metallic flat plate which only re-directs the wave back in the same direction at normal incidence which is $6 \mathrm{~dB}$ in this case. The gain $(\mathrm{dB})$ of monostatic RCS for each incident angle of the optimized metasurface compared to that of the mettalic flat plate are given in Table. 2. Some angles such as that at $+20.3^{\circ}$, are subject to more attenuation due to the surface resistance. In terms of frequency and polarization, the metasurface is said to operate at $14.7 \mathrm{GHz}$, as well as a tolerance of $\pm 100 \mathrm{MHz}$ around the operating frequency. On the other hand, for applications where broadband and dual polarization is needed other unit cell designs can be considered $[12,15]$.

Table 2 Gain in dB of monostatic RCS of the optimized metasurface compared to that of a flat metallic plate of similar dimensions $5 \mathrm{~cm} \times 23 \mathrm{~cm}$ for the given incident angles $\theta_{i}$ at $14.7 \mathrm{GHz}$.

\begin{tabular}{cc}
$\theta_{i}{ }^{\circ}$ & Gain $(\mathrm{dB})$ \\
\hline-60 & +17 \\
-44 & +23 \\
-32 & +22 \\
-20.3 & +21 \\
-10 & +32 \\
0 & -4 \\
+10 & +32 \\
+20.3 & +23 \\
+32 & +24 \\
+44 & +27 \\
+60 & +21
\end{tabular}

\section{Experimental Measurements}

To validate the feasibility of our metasurface design, a prototype of the optimized metasurface of $10 \times 7$ supercells and dimensions $10.2 \mathrm{~cm} \times 41.125 \mathrm{~cm}$ has been fabricated. The surface design has been printed on top of a Roger Duroid 5880 grounded substrate of dielectric constant $\epsilon=2.2$ and thickness $1.6 \mathrm{~mm}$. The prototype is shown in Fig.9. The measurements were carried out in an anechoic chamber using two vivaldi antennas, one acting as a transmitter and the other as a receiver. The antennas were place next to each other and isolated using absorbing material to eliminate the coupling. The operating frequency of the antennas was $14.7 \mathrm{GHz}$. The metasurface design was placed on top of a rotating mechanical platform and both antennas were fixed at a distance of 3 meters from the target. The far-field monostatic RCS has been calculated. Since the metasurface prototype used for measurements was of larger dimensions compared to that of simulated design (for less computational time analysis), the normalized monostatic RCS of the measurements have been compared to that of the simulation results in order to highlight the performance of the design in terms of retro-reflection at each angle of incidence as given in Fig.10.

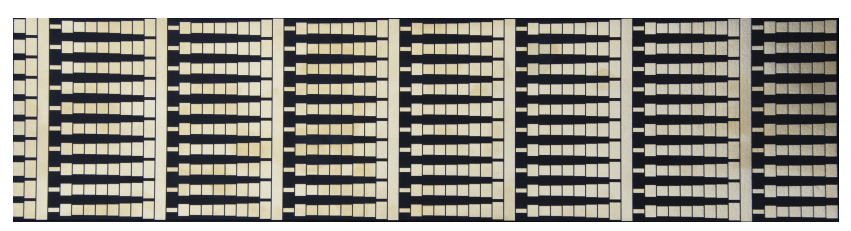

Fig. 9 Realized Metasurface prototype of $10 \times 7$ super cells of dimensions $10.2 \mathrm{~cm} \times 41.125 \mathrm{~cm}$.

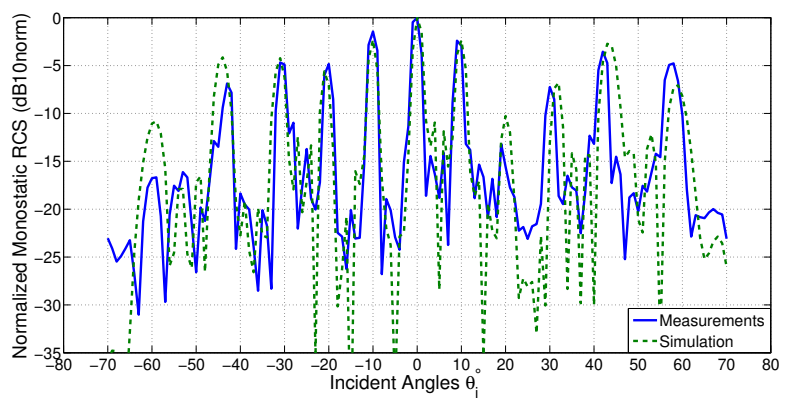

Fig. 10 Normalized Monostatic RCS (dB10norm) with respect to the incident angles of the simulation results compared to that of the measurements at $14.7 \mathrm{GHz}$ for the optimized metasurface.

The experimental measurement have shown very good agreement compared to that of the simulation results and good performance in terms of retro-reflection of 
the optimized metasurface at multiple incident angles $\left( \pm 10^{\circ}, \pm 20.3^{\circ}, \pm 32^{\circ}, \pm 44^{\circ}, \pm 60^{\circ}\right.$ and normal incidence $)$ as shown in Fig.10.

\section{Conclusion}

In this paper, we demonstrated the method and design to retain the conventional properties for a retrodirective metasurface in the reciprocal direction and at normal incidence for high super-cell periodicity designs beyond $2 \lambda$. The method is based on the generalized phase law of reflection and the impedance modulation technique. Instead of using the cascading method, with a well engineered surface impedance using one single super-cell design, it can be more reliable and efficient to achieve retrodirectivity in the three desired directions. As well as, according to Floquet Bloch analysis, higher orders of diffraction exist at these periodicities making the retrodirective metasurface design more lossy in terms of retro-reflection for the propagating electromagnetic wave. Thus, these diffraction orders can be controlled by optimizing the surface impedance to achieve efficient retro-reflection at multiple incident directions simultaneously.

With an increase of periodicity with respect to $\lambda$, a wide number of diffraction orders can exist, thus yielding to retrodirectivity simultaneously at a large number of incident angles, with a well optimized surface impedance super-cell design. This is an interesting feature, where a single compact metasurface design can achieve retro-reflection for a broadband span of angles with high efficiency. Furthermore, this can be significant for different applications in physical optics, which give exceptional physical properties in terms of light manipulation, and at microwave frequencies such as RCS enhancement specially for targets where backscattering is poor.

\section{Acknowledgments}

This work has been carried out in the context of the French national project CYCLOPE funded by Agence national de la recherche and the regional project ORIO in the framework of the ELSAT 2020 program co-financed by the European Union with the European Regional development fund, the French state and Hauts de France Regional council.

\section{References}

1. H.T. Chen, A.J. Taylor, N. Yu, Reports on Progress in Physics 79(7), 076401 (2016)
2. S.B. Glybovski, S.A. Tretyakov, P.A. Belov, Y.S. Kivshar, C.R. Simovski, Physics reports 634, 1 (2016)

3. S. Sun, K.Y. Yang, C.M. Wang, T.K. Juan, W.T. Chen, C.Y. Liao, Q. He, S. Xiao, W.T. Kung, G.Y. Guo, L. Zhou, D.P. Tsai, Nano Letters 12(12), 6223 (2012). DOI 10.1021/nl3032668. URL http://dx.doi.org/10.1021/nl3032668. PMID: 23189928

4. C.L. Holloway, E.F. Kuester, J.A. Gordon, J. O'Hara, J. Booth, D.R. Smith, IEEE Antennas and Propagation Magazine 54(2), 10 (2012). DOI 10.1109/MAP.2012.6230714

5. A. Pors, M.G. Nielsen, R.L. Eriksen, S.I. Bozhevolnyi, Nano letters 13(2), 829 (2013)

6. A. Pors, S.I. Bozhevolnyi, Optics express 21(22), 27438 (2013)

7. A. Pors, O. Albrektsen, I.P. Radko, S.I. Bozhevolnyi, Scientific reports $\mathbf{3}(2155), 1$ (2013)

8. L. Zhang, S. Mei, K. Huang, C.W. Qiu, $\begin{array}{llll}\text { Advanced Optical Materials 4(6), } 818 & \end{array}$ (2016). DOI 10.1002/adom.201500690. URL http://dx.doi.org/10.1002/adom.201500690

9. A.A. Elsakka, V.S. Asadchy, I.A. Faniayeu, S.N. Tcvetkova, S.A. Tretyakov, IEEE Transactions on Antennas and Propagation 64(10), 4266 (2016)

10. C. Pfeiffer, A. Grbic, Applied Physics Letters 102(23), 231116 (2013)

11. G. Zheng, H. Mühlenbernd, M. Kenney, G. Li, T. Zentgraf, S. Zhang, Nature nanotechnology 10(4), 308 (2015)

12. M. Kalaagi, D. Seetharamdoo, in 2018 IEEE Radio and Antenna Days of the Indian Ocean (RADIO) (IEEE, 2018), pp. 1-2

13. O. Yurduseven, D.R. Smith, IEEE Antennas and Wireless Propagation Letters 16, 2738 (2017)

14. A. Cala'Lesina, L. Ramunno, P. Berini, Optics letters 40(12), 2874 (2015)

15. Z. Chen, H. Deng, Q. Xiong, C. Liu, Applied Physics A 124(3), 281 (2018)

16. L. Liu, X. Zhang, M. Kenney, X. Su, N. Xu, C. Ouyang, Y. Shi, J. Han, W. Zhang, S. Zhang, Advanced materials 26(29), 5031 (2014)

17. Z. Li, E. Palacios, S. Butun, K. Aydin, Nano letters 15(3), 1615 (2015)

18. E.F. Knott, Radar cross section measurements (Springer Science \& Business Media, 2012)

19. Y.C. Guo, X.W. Shi, L. Chen, Progress In Electromagnetics Research B 5, 153 (2008)

20. N. Yu, P. Genevet, M.A. Kats, F. Aieta, J.P. Tetienne, F. Capasso, Z. Gaburro, science 334(6054), 333 (2011)

21. E. Doumanis, G. Goussetis, G. Papageorgiou, V. Fusco, R. Cahill, D. Linton, IEEE Transactions on Antennas and Propagation 61(1), 232 (2013)

22. C. Shen, A. Díaz-Rubio, J. Li, S.A. Cummer, Applied Physics Letters 112(18), 183503 (2018)

23. M. Kalaagi, D. Seetharamdoo, Journal of Applied Physics 126(10), 104901 (2019)

24. A. Epstein, G.V. Eleftheriades, Physical Review B 90(23), $235127(2014)$

25. V. Asadchy, A. Díaz-Rubio, S. Tcvetkova, D.H. Kwon, A. Elsakka, M. Albooyeh, S. Tretyakov, Physical Review $\mathrm{X} \mathbf{7}(3), 031046$ (2017)

26. V. Asadchy, M. Albooyeh, S. Tcvetkova, A. Díaz-Rubio, Y. Ra'di, S. Tretyakov, Physical Review B 94(7), 075142 (2016) 